\title{
CONFORMAL MEASURES FOR MEROMORPHIC MAPS
}

\author{
Krzysztof Barański, Bogusława Karpińska and Anna Zdunik \\ University of Warsaw, Institute of Mathematics \\ ul. Banacha 2, 02-097 Warszawa, Poland; baranski@mimuw.edu.pl \\ Warsaw University of Technology, Faculty of Mathematics and Information Science \\ ul. Koszykowa 75, 00-662 Warszawa, Poland; bkarpin@mini.pw.edu.pl \\ University of Warsaw, Institute of Mathematics \\ ul. Banacha 2, 02-097 Warszawa, Poland; A.Zdunik@mimuw.edu.pl
}

\begin{abstract}
In this paper we study relations between the existence of a conformal measure on the Julia set $J(f)$ of a transcendental meromorphic map $f$ and the existence of a zero of the topological pressure function $t \mapsto P(f, t)$ for the map $f$ (with respect to the spherical metric). In particular, we show that if $f$ is hyperbolic and admits a $t$-conformal measure which is not totally supported on the set of escaping points of $f$, then $P(f, t)=0$. On the other hand, for a wide class of maps $f$, including arbitrary maps with at most finitely many poles and finite set of singular values as well as hyperbolic maps with at most finitely many poles, if $P(f, t)=0$, then there exists a $t$-conformal measure on $J(f)$. This partially answers a question of Mauldin.
\end{abstract}

\section{Introduction}

Let $f: \mathbf{C} \rightarrow \widehat{\mathbf{C}}$ be a transcendental meromorphic function. We denote by $f^{n}=$ $f \circ \cdots \circ f$ the $n$-th iterate of $f$. The Fatou set $F(f)$ consists of all points $z \in \mathbf{C}$ for which there exists a neighbourhood $U$ of $z$ such that the family of iterates $\left\{\left.f^{n}\right|_{U}\right\}_{n>0}$ is defined and normal. The complement $\mathbf{C} \backslash F(f)$ is called the Julia set and is denoted by $J(f)$. Note that some authors define $J(f)$ as $\widehat{\mathbf{C}} \backslash F(f)$, so that it always contains the point at infinity. In this paper we adopt the convention $\infty \notin J(f)$. Intuitively, the Julia set carries the chaotic part of the dynamics of $f$. See e.g. [Ber93] for a detailed presentation of the theory of iteration of transcendental meromorphic maps.

In this paper we investigate ergodic properties of the dynamics of transcendental maps, using tools of the thermodynamic formalism, developed by Ruelle, Bowen and Walters in the 1970's and applied successfully to the study of the dynamics of rational maps on the Riemann sphere (see [Rue78, PU10] and the references therein). An important result in this area is the celebrated Bowen's formula (see [Bow79]), which states that the Hausdorff dimension of the Julia set $J(f)$ of a hyperbolic rational map $f$ of degree $d \geq 2$ is equal to the unique zero of the topological pressure function

$$
\mathbf{R}_{+} \ni t \mapsto \lim _{n \rightarrow \infty} \frac{1}{n} \ln \sum_{w \in f^{-n}\left(z_{0}\right)}\left|\left(f^{n}\right)^{\prime}(w)\right|^{-t}
$$

for $z_{0} \in J(f)$. Note that in general, the topological pressure is defined with limsup instead of lim in the above formula. However, in the considered case the limit in (1) exists (see [PU10, Remark 12.5.8]. Recall that a rational map $f$ is hyperbolic, if the

https://doi.org/10.5186/aasfm.2018.4329

2010 Mathematics Subject Classification: Primary 37F10, 37F35; Secondary 28A80.

Key words: Meromorphic functions, Julia sets, conformal measures, topological pressure.

Research supported by the Polish NCN grant 2014/13/B/ST1/04551, carried out at the University of Warsaw. 
closure in $\widehat{\mathbf{C}}$ of the union of forward trajectories of all critical values of $f$ is disjoint from $J(f)$.

Attempts to generalise the theory of thermodynamic formalism to the case of transcendental meromorphic maps started in the 1990's (see [Bar95, MU96]) and progressed in the subsequent years (see e.g. [KU02, UZ03, KU04, UZ04, KU05, KU06, CS07, UZ07, MU10, BKZ09, BKZ12, May17] and surveys [KU08, MU10]).

In [UZ03, UZ04], Urbański and Zdunik established the thermodynamic formalism theory for hyperbolic exponential maps of the form $z \mapsto \lambda e^{z}, \lambda \in \mathbf{C} \backslash\{0\}$. Recall that for the transcendental case, in the definition of a hyperbolic map $f$ one requires that the closure of the union of forward trajectories of all singular (critical and asymptotic) values of $f$ is bounded and disjoint from the Julia set (see Definition 2.1). In the above two papers the authors discovered a crucial role of the radial Julia set $J_{r}(f)$ (see Definition 2.3) in the study of ergodic properties of transcendental maps. In particular, it turned out that the Bowen formula holds for a large class of transcendental meromorphic maps in a modified form: the zero of the pressure function is equal to the Hausdorff dimension of the radial Julia set. As shown in [Rem09], the dimension of the radial Julia set coincides with the hyperbolic dimension of $J(f)$. Contrary to the case of rational dynamics, the radial Julia set of a transcendental map is often essentially smaller (in the sense of dimension) than the whole Julia set, even in the hyperbolic case. For instance, the Julia set of an arbitrary exponential map has Hausdorff dimension 2 (see [McM87]), while the Hausdorff dimension of the radial Julia set for a hyperbolic exponential map is greater than 1 and smaller than 2 (see [UZ03, UZ04]). The fact that the Hausdorff dimension of $J_{r}(f)$ is greater than 1 was generalised in [BKZ09] to the case of transcendental meromorphic maps with logarithmic tracts over $\infty$ (see Definition 2.6), in particular, for maps with a bounded set of singular values $($ class $\mathcal{B})$, which are entire or meromorphic with a finite number of poles.

In [Bar95, KU02, KU04, KU05, CS07], some elements of the thermodynamic formalism theory were established for other families of hyperbolic transcendental maps, both entire and meromorphic, including the sine and tangent family. The most general approach was presented in [MU10, MU10], where Mayer and Urbański developed a detailed theory for hyperbolic transcendental meromorphic maps of finite order with the balanced derivative growth condition, which relates the growth of the derivative of the function (at a point tending to infinity) with the growth of the function itself. This class includes many families of maps of the form $Q\left(e^{R}\right)$, where $Q, R$ are polynomials or rational functions.

Note that the pressure function defined in (1) with the derivative of $f$ in the standard (Euclidean) metric is usually not suitable in transcendental case, since it can be infinite for all values of $t$. To overcome this difficulty, one considers derivative in some other conformal metric on C. For instance, in [MU10, MU10], this metric has the form $|d \rho|=\frac{|d z|}{1+|z|^{\beta}}$ for a suitable $\beta \in \mathbf{R}$.

In the previous paper [BKZ12] the authors considered the topological pressure function

$$
P(f, t)=P\left(f, t, z_{0}\right)=\lim _{n \rightarrow \infty} \frac{1}{n} \ln \sum_{w \in f^{-n}\left(z_{0}\right)}\left|\left(f^{n}\right)^{*}(w)\right|^{-t}
$$

for $t>0$, where $f^{*}$ is the derivative of $f$ taken with respect to the spherical metric (see Section 2 for details). They proved that for all transcendental meromorphic maps with a finite set of singular values $($ class $\mathcal{S}$ ) and a large family of maps with 
a bounded set of singular values (class $\mathcal{B}$ ), including all hyperbolic transcendental meromorphic maps, the pressure defined in (2) exists and does not depend on typical $z_{0}$. In addition, in [BKZ12] they present a version of Bowen's formula for such maps, which asserts that the Hausdorff dimension of the radial Julia set of a map $f$ is equal to a number $t_{0}$, defined as the infimum of the values $t>0$ for which $P(f, t)$ is nonpositive. See Definition 2.9 and Theorem 2.10 for a precise formulation. Note that similar results were obtained previously by Przytycki, Rivera-Letelier and Smirnov [Prz99, PRLS04] for arbitrary rational maps of degree $d \geq 2$.

In the present paper we consider another element of the thermodynamic formalism theory in a general setup of transcendental maps, investigating the question of the existence of a $t$-conformal measure, i.e. a Borel probability measure $\nu$ on $J(f)$ such that

$$
\nu(f(A))=\int_{A}\left|f^{\prime}(z)\right|^{t} d \nu(z)
$$

for every Borel set $A \subset \mathbf{C}$ on which $f$ is injective (see Definition 2.4). This notion, introduced by Patterson [Pat76] and Sullivan [Sul82]) in the context of Fuchsian and Kleinian groups, and developed in the papers by Denker and Urbański (see e.g. [DU91a, DU91b, DU91c]), proved to be extremely useful in many areas of conformal dynamics (see e.g. [LSV98, DG99, Prz99, BPS01, GS09, VV10, Tho12] and surveys [Urb03, KU08]).

In the context of rational dynamics, it is known that the Julia set of a rational map always admits a $t$-conformal measure for a suitable $t>0$, and the minimal exponent $t$ for which such a measure exists is equal to the Hausdorff dimension of the radial Julia set (see e.g. [Prz99]). In the case of transcendental maps the question of existence of a $t$-conformal measure, where $t$ is the Hausdorff dimension of the radial Julia set, was answered positively for several specific families of maps, e.g. for the maps considered in [UZ07, MU10]. However, the general question on existence of such a conformal measure has been open.

Seeking analogies between transcendental dynamics and the theory of infinite conformal iterated function systems (CIFS), developed by Mauldin and Urbański (see [MU96]), recall that a conformal iterated function system is called regular, if it admits a conformal measure on its limit set. In [MU96, Theorem 3.5 and Lemma $3.13]$ it was proved that a CIFS is regular if and only if there exists $t>0$ such that $P(t)=0$ where $P(t)$ is the topological pressure function defined for this system. In view of this, Mauldin (in a private communication) asked the following question.

Question. Let $f: \mathbf{C} \rightarrow \widehat{\mathbf{C}}$ be a transcendental meromorphic function for which the pressure function $P(f, t)$ defined in (2) exists. Is the existence of a value $t>0$ such that $P(f, t)=0$ equivalent to the existence of a $t$-conformal measure on $J(f)$ ?

Here and in the sequel a $t$-conformal measure on $J(f)$ is taken with respect to the spherical metric and denoted by $m_{t}$, i.e. satisfies

$$
m_{t}(f(A))=\int_{A}\left|f^{*}(z)\right|^{t} d m_{t}(z)
$$

for every Borel set $A \subset \mathbf{C}$ on which $f$ is injective.

Note that, in general, we do not know in which cases we have $P\left(f, t_{0}\right)=0$ for $t_{0}$ defined in (2) (although $P(f, t)$ is continuous and convex when it is finite, it could have a 'jump' from the infinite value at $\left.t=t_{0}\right)$. It is known that $P\left(f, t_{0}\right)=0$ for some classes of maps $f$ considered in [MU10, MU10], but it is an open question whether the opposite case can actually appear (cf. Proposition 3.8). 
In this paper we partially answer Mauldin's question. The first result we prove is the following.

Theorem A. If a hyperbolic transcendental meromorphic map $f: \mathbf{C} \rightarrow \widehat{\mathbf{C}}$ admits a $t$-conformal measure $m_{t}$ on $J(f)$ for some $t>0$, with respect to the spherical metric, then $P(f, t) \leq 0$. Moreover, if $m_{t}(J(f) \backslash I(f))>0$, then $P(f, t)=0$.

By $I(f)$ we denote the escaping set of $f$ defined as

$$
I(f)=\left\{z \in \mathbf{C}:\left\{f^{n}(z)\right\}_{n=0}^{\infty} \text { is defined and } f^{n}(z) \rightarrow \infty \text { as } n \rightarrow \infty\right\} .
$$

The case $m_{t}(J(f) \backslash I(f))=0$ in Theorem A can actually occur, as shown in the following example.

Example 1.1. For $f(z)=\lambda \sin z, \lambda \in \mathbf{C} \backslash\{0\}$. Then $I(f)$ has positive 2dimensional Lebesgue measure (see [McM87]) and the normalized 2-dimensional spherical Lebesgue measure on $I(f)$ is 2-conformal. Moreover, if additionally, $f$ is hyperbolic, then $P(f, 2)<0$ (see [CS07, MU10]).

Theorem A is an immediate corollary of the following more general result.

Theorem B. Let $f: \mathbf{C} \rightarrow \widehat{\mathbf{C}}$ be a transcendental meromorphic map. Assume that there exists a $t$-conformal measure $m_{t}$ on $J(f)$ for some $t>0$, with respect to the spherical metric.

(a) Suppose $f \in \mathcal{S}$. Then at least one of the following two assertions hold. $\circ P(f, t) \leq 0$.

- The measure $m_{t}$ is totally supported on a set $E \subset \overline{\mathcal{P}(f)} \cap J(f)$ of Hausdorff dimension 0.

Moreover, at least one of the following two assertions hold.

○ $P(f, t) \geq 0$.

○ $f^{n}(z) \rightarrow(\overline{\mathcal{P}(f)} \cap J(f)) \cup\{\infty\}$ as $n \rightarrow \infty$ for $m_{t}$-almost every $z$.

(b) Suppose $f \in \mathcal{B}$, such that $J(f) \backslash \overline{\mathcal{P}(f)} \neq \emptyset$ and $J(f)$ does not contain (Picard) exceptional values of $f$. Then $P(f, t) \leq 0$. Moreover, at least one of the following two assertions hold.

○ $P(f, t)=0$.

○ $f^{n}(z) \rightarrow(\overline{\mathcal{P}(f)} \cap J(f)) \cup\{\infty\}$ as $n \rightarrow \infty$ for $m_{t}$-almost every $z$.

Remark 1.2. In fact, in the case (b) the assumptions can be weakened. It is enough to assume that the map $f \in \mathcal{B}$ is non-exceptional (see Definition 2.2), $J(f) \backslash \overline{\mathcal{P}(f)} \neq \emptyset$ and $J(f)$ contains no parabolic cycles of length 1 or 2 and multiplier 1 composed of exceptional values of $f$, nor exceptional values which are non-fixed critical points of $f$ (including poles of $f$ of degree larger than 1 ).

The next result we prove in this paper assumes that the map $f$ has a logarithmic tract over $\infty$ (see Definition 2.6). See also Definition 2.2 for the definition of exceptional maps.

Theorem C. Let $f: \mathbf{C} \rightarrow \widehat{\mathbf{C}}$ be a transcendental meromorphic map with a logarithmic tract over $\infty$. Assume that $f$ is an arbitrary map from class $\mathcal{S}$, or a non-exceptional map from class $\mathcal{B}$ such that $J(f) \backslash \overline{\mathcal{P}(f)} \neq \emptyset$. If $P(f, t)=0$ for some $t>0$, then there exists a $t$-conformal measure $m_{t}$ on $J(f)$, with respect to the spherical metric. Moreover,

$$
m_{t}(\mathbf{C} \backslash \mathbf{D}(r))=o\left(\frac{(\ln r)^{3 t}}{r^{t}}\right) \quad \text { as } \quad r \rightarrow \infty,
$$


where $\mathbf{D}(r)=\{z \in \mathbf{C}:|z|<r\}$.

In particular, the assertions hold for all hyperbolic transcendental meromorphic maps $f$, which are entire or have a finite number of poles.

Remark 1.3. In fact, the proof gives

$$
\int_{1}^{\infty} \frac{r^{t}}{(\ln r)^{3 t}} m_{t}(\mathbf{C} \backslash \mathbf{D}(r)) d r<\infty
$$

Remark 1.4. Under the assumptions of Theorem C, for every $\alpha>1 / 2$, the probability measure $m_{t}^{(\alpha)}$ on $J(f)$ defined as $d m_{t}^{(\alpha)}=\eta d m_{t} / \int \eta d m_{t}$ for $\eta(z)=$ $\left(1+|z|^{2}\right)^{t} /\left(1+|z|^{\alpha}\right)^{t}$ is $t$-conformal with respect to the conformal metric $|d z| /\left(1+|z|^{\alpha}\right)$. The case $\alpha=1$, concerning the 'cylindrical' metric is of special interest, since it corresponds to the situation, when the map is lifted by the logarithmic coordinates, which is now a standard tool in transcendental dynamics.

Remark 1.5. Theorems A, B and C contribute to answer the Mauldin question in both directions. A main problem which remains to be determined, is under which conditions the measure $m_{t}$ constructed in Theorem $\mathrm{C}$ satisfies $m_{t}(J(f) \backslash I(f))=0$.

The plan of the paper is as follows. After preliminary Section 2, containing notation, basic definitions and used results, in Section 3 we prove some general facts concerning the radial Julia sets, conformal measures and topological pressure. The proof of Theorem B and Remark 1.2 is presented in Section 4 (as noted above, this proves also Theorem A). The last Section 5 contains the proof of Theorem $\mathrm{C}$ together with Remarks 1.3 and 1.4 .

Acknowledgement. We wish to thank the referees for helpful comments and suggestions.

\section{Preliminaries}

Notation. In all definitions and formulations of the results we assume that $f$ is a transcendental meromorphic function on the complex plane.

By a conformal metric we mean a Riemannian metric on $\mathbf{C}$ of the form

$$
|d \rho|=\rho|d z|,
$$

where $|d z|$ is the standard (Euclidean) metric and $\rho$ is a continuous positive function on $\mathbf{C}$. The derivative of a map $f$ with respect to the metric $d \rho$ is equal to

$$
f_{\rho}^{\prime}(z)=\frac{\rho(f(z))}{\rho(z)} f^{\prime}(z)
$$

where $f^{\prime}$ is the standard derivative. In particular, we consider the spherical metric defined by

$$
|d s|=\frac{2|d z|}{1+|z|^{2}}
$$

and the spherical derivative

$$
f^{*}(z):=f_{s}^{\prime}(z)=\frac{\left(1+|z|^{2}\right) f^{\prime}(z)}{1+|f(z)|^{2}} .
$$

The spherical distance in $\widehat{\mathbf{C}}$ (defined by the spherical metric) will be denoted by dist $_{s p h}$. 
By $\mathbf{D}(z, r)$ (resp. $\mathcal{D}(z, r))$ we denote the disc centred at $z \in \mathbf{C}($ resp. $z \in \widehat{\mathbf{C}})$ of radius $r>0$ with respect to the Euclidean (resp. spherical) metric. For simplicity, we write $\mathbf{D}(r)$ for $\mathbf{D}(0, r)$.

Definition 2.1. We write $\operatorname{Sing}(f)$ for the singular set of $f$, which consists of all finite singular (critical and asymptotic) values of $f$ and define the post-singular set by

$$
\mathcal{P}(f)=\bigcup_{n=0}^{\infty} f^{n}(\operatorname{Sing}(f)),
$$

where we neglect terms which are not defined.

Recall that we consider two classes of transcendental meromorphic functions:

$$
\mathcal{S}=\{f: \operatorname{Sing}(f) \text { is finite }\}, \quad \mathcal{B}=\{f: \operatorname{Sing}(f) \text { is bounded }\} .
$$

The class $\mathcal{B}$ was introduced in the context of holomorphic dynamics by Eremenko and Lyubich in [EL92], and $\mathcal{S}$ is called the Speiser class.

We say that $f$ is hyperbolic, if $\overline{\mathcal{P}(f)}$ is bounded and disjoint from the Julia set of $f$. Note that hyperbolic maps are in the class $\mathcal{B}$.

Definition 2.2. We call $f$ exceptional, if there exists a (Picard) exceptional value $a$ of $f$, such that $a \in J(f)$ and $f$ has a non-logarithmic singularity over $a$.

\section{Radial Julia sets and conformal measures.}

Definition 2.3. The radial Julia set $J_{r}(f)$ is the set of points $z \in J(f)$ for which all iterates $f^{n}(z)$ are defined and there exist $r>0$ and a sequence $n_{k} \rightarrow \infty$, such that a holomorphic inverse branch of $f^{n_{k}}$ sending $f^{n_{k}}(z)$ to $z$ is well-defined on the spherical disc $\mathcal{D}\left(f^{n_{k}}(z), r\right)$.

Recall that we denote by $I(f)$ the escaping set of $f$, i.e.

$$
I(f)=\left\{z \in \mathbf{C}:\left\{f^{n}(z)\right\}_{n=0}^{\infty} \text { is defined and } f^{n}(z) \rightarrow \infty \text { as } n \rightarrow \infty\right\} .
$$

We consider conformal measures with respect to some conformal metrics on $\mathbf{C}$.

Definition 2.4. We say that a Borel probability measure $\nu$ on $J(f)$ is $t$-conformal for some $t>0$, with respect to a conformal metric $|d \rho|=\rho|d z|$, if

$$
\nu(f(A))=\int_{A}\left|f_{\rho}^{\prime}(z)\right|^{t} d \nu(z)
$$

for every Borel set $A \subset \mathbf{C}$ on which the map $f$ is injective.

As noted in the introduction, in this paper we consider $t$-conformal measures taken with respect to the spherical metric.

Distortion estimates. We use the following spherical version of the classical Koebe Distortion Theorem, see, e.g. [BKZ12] for its detailed proof.

Theorem 2.5. (Spherical Distortion Koebe Theorem) Let $0<r_{1}, r_{2}<\operatorname{diam}_{s p h} \widehat{\mathbf{C}}$. Then there exists a constant $c>0$ depending only on $r_{1}, r_{2}$, such that for every spherical disc $D=\mathcal{D}\left(z_{0}, r\right)$ and every univalent holomorphic map $g: D \rightarrow \widehat{\mathbf{C}}$ with $z_{0} \in \widehat{\mathbf{C}}$, $\operatorname{diam}_{s p h} D<r_{1}$ and $\operatorname{diam}_{s p h}(\widehat{\mathbf{C}} \backslash g(D))>r_{2}$, if $z_{1}, z_{2} \in \mathcal{D}\left(z_{0}, \lambda r\right)$ for some $0<\lambda<1$, then

$$
\frac{\left|g^{*}\left(z_{1}\right)\right|}{\left|g^{*}\left(z_{2}\right)\right|} \leq \frac{c}{(1-\lambda)^{4}}
$$

We recall the notion of a logarithmic tract, and formulate some distortion estimates which will be used in subsequent sections. 
Definition 2.6. Let $U \subset \mathbf{C}$ be an unbounded simply connected domain, such that the boundary of $U$ in $\mathbf{C}$ is a smooth open simple arc, and let $R>0$. If $f: \bar{U} \rightarrow \mathbf{C}$ is a continuous map, such that $|f(z)|=R$ for every $z$ in the boundary of $U$ and $f$ on $U$ is a holomorphic universal covering of $\{z \in \mathbf{C}:|z|>R\}$, then we call $U$ a logarithmic tract of $f$ over $\infty$.

Remark 2.7. If a map $f \in \mathcal{B}$ is entire or has a finite number of poles, and $R$ is sufficiently large, then there exists a component of $f^{-1}(V)$, where $V=\{z \in \mathbf{C}:|z|>$ $R\}$, which is a logarithmic tract of $f$ over $\infty$. See [EL92] for details.

We shall make use of the following facts, proved in [BKZ12, Corollaries 3.7 and 3.9]. They strengthen the estimates proved previously in [Sta99, Lemmas 2.6 and $2.7]$.

Lemma 2.8. Let $R, L>1$. Then there exist constants $c_{1}, c_{2}>0$ depending only on $R, L$, such that for every logarithmic tract $U \subset \mathbf{C}$ of a map $f: U \rightarrow V$ over $\infty$, where $V=\{z \in \mathbf{C}:|z|>R\}$ and $0 \notin U$, for every $z_{1}, z_{2} \in V$ with $\left|z_{1}\right| \geq\left|z_{2}\right| \geq L R$ and every inverse branch of $f$ in a neighbourhood of $z_{1}$ (resp. $\left.z_{2}\right)$, denoted by $g$, we have

$$
\begin{gathered}
c_{1}^{-1}\left(\frac{\ln \left|z_{1}\right|}{\ln \left|z_{2}\right|}\right)^{-4 \pi}<\frac{\left|g\left(z_{1}\right)\right|}{\left|g\left(z_{2}\right)\right|}<c_{1}\left(\frac{\ln \left|z_{1}\right|}{\ln \left|z_{2}\right|}\right)^{4 \pi}, \\
c_{2}^{-1} \frac{\left|z_{1}\right|}{\left|z_{2}\right|}\left(\frac{\ln \left|z_{1}\right|}{\ln \left|z_{2}\right|}\right)^{-3} \leq \frac{\left|g^{*}\left(z_{1}\right)\right|}{\left|g^{*}\left(z_{2}\right)\right|} \leq c_{2} \frac{\left|z_{1}\right|}{\left|z_{2}\right|} \frac{\ln \left|z_{1}\right|}{\ln \left|z_{2}\right|},
\end{gathered}
$$

for some extension of the branch $g$ to a neighbourhood of $z_{2}$ (resp. $z_{1}$ ).

Pressure for transcendental maps.

Definition 2.9. The topological pressure function with respect to the spherical metric is defined as

$$
P\left(f, t, z_{0}\right)=\lim _{n \rightarrow \infty} \frac{1}{n} \ln \sum_{w \in f^{-n}\left(z_{0}\right)}\left|\left(f^{n}\right)^{*}(w)\right|^{-t}
$$

for $z_{0} \in \mathbf{C}$ and $t>0$, assuming that the limit exists (possibly infinite).

We use the following results, proved in [BKZ12], establishing the existence of the pressure function and Bowen's formula for transcendental meromorphic maps.

Theorem 2.10. [BKZ12, Theorems A and B] For every transcendental entire or meromorphic map $f$ in the class $\mathcal{S}$ and every $t>0$ the topological pressure $P(f, t)=P\left(f, t, z_{0}\right)$ exists (possibly equal to $+\infty$ ) and is independent of $z_{0} \in \mathbf{C}$ up to an exceptional set of Hausdorff dimension zero (consisting of points quickly approximated by the forward orbits of singular values of $f$ ). We have

$$
P(f, t)=P_{h y p}(f, t),
$$

where $P_{\text {hyp }}(f, t)$ is the supremum of the pressures $P\left(\left.f\right|_{X}, t\right)$ over all transitive isolated conformal repellers $X \subset J(f)$. The function $t \mapsto P(f, t)$ is non-increasing and convex when it is finite. In addition, we have $P(f, 2) \leq 0$. The following version of Bowen's formula holds:

$$
\operatorname{dim}_{H} J_{r}(f)=\operatorname{dim}_{h y p} J(f)=t_{0},
$$

where $t_{0}=\inf \{t>0: P(f, t) \leq 0\}$. 
Moreover, analogous results hold for every non-exceptional transcendental entire or meromorphic map $f$ in the class $\mathcal{B}$, such that $J(f) \backslash \overline{\mathcal{P}(f)} \neq \emptyset$ (in particular, for every hyperbolic map) and $z_{0}$ in $J(f) \backslash \overline{\mathcal{P}(f)}$, which is an open dense subset of $J(f)$.

If $f$ is hyperbolic, then $P(f, t)>0$ (possibly equal to $+\infty$ ) for every $0<t<t_{0}$ and $P(f, t)<0$ for every $t>t_{0}$.

We refer to the points $z_{0} \in \mathbf{C}$ outside the exceptional set from Theorem 2.10 as to GPS points (good pressure starting points).

Following [BKZ12], for a set $A \subset \mathbf{C}$ and $n>0$ we denote

$$
S_{n}(t, z)=\sum_{w \in f^{-n}(z)}\left|\left(f^{n}\right)^{*}(w)\right|^{-t}, \quad S_{n}^{A}(t, z)=\sum_{w \in f^{-n}(z) \cap A}\left|\left(f^{n}\right)^{*}(w)\right|^{-t} .
$$

It is understood that a sum over an empty set is zero.

\section{Introductory results}

In this section we present some results concerning the properties of the radial Julia set, conformal measures and topological pressure. Note that some facts are not used in the subsequent sections but are of independent interest. In all results of this section we assume that $f$ is a transcendental meromorphic map on the complex plane.

The first two propositions study properties of the radial Julia set $J_{r}(f)$ and the set of non-escaping points in the Julia set.

Proposition 3.1. $\quad$ (a) If $f$ has a finite number of poles, then $J_{r}(f) \subset J(f) \backslash$ $\left(I(f) \cup \bigcup_{n=1}^{\infty} f^{-n}(\infty)\right)$. In particular, if $f$ is entire, then $J_{r}(f) \subset J(f) \backslash I(f)$.

(b) If $f$ is hyperbolic, then $J(f) \backslash\left(I(f) \cup \bigcup_{n=1}^{\infty} f^{-n}(\infty)\right) \subset J_{r}(f)$. In particular, if $f$ is hyperbolic entire, then $J_{r}(f)=J(f) \backslash I(f)$.

Proof. First, note that by definition, $J_{r}(f) \cup(I(f) \cap J(f)) \subset J(f) \backslash \bigcup_{n=1}^{\infty} f^{-n}(\infty)$.

To show (a), assume that $f$ has a finite number of poles and suppose that $z \in$ $J_{r}(f) \cap I(f)$. Then there exist $r>0$ and a sequence $n_{k} \rightarrow \infty$, such that the inverse branch of $f$ sending $f^{n_{k}}(z)$ to $f^{n_{k}-1}(z)$, denoted by $g_{k}$, is defined on $\mathcal{D}\left(f^{n_{k}}(z), r\right)$. Since $z \in I(f)$, we have $\infty \in \mathcal{D}\left(f^{n_{k}}(z), r / 2\right)$ for large $k$, which implies that $g_{k}$ is defined on $\mathcal{D}(\infty, r / 2)$, sending $\infty$ to some pole $p_{k}$ of $f$. Since the number of poles is finite, passing to a subsequence we can assume that $p_{k} \equiv p$ for some pole $p$. Then $f^{n_{k}-1}(z)=g_{k}\left(f^{n_{k}}(z)\right)$ is in a small neighbourhood of $p$ for every large $k$, which contradicts $z \in I(f)$.

To prove (b), suppose $f$ is hyperbolic and take $z \in J(f) \backslash\left(I(f) \cup \bigcup_{n=1}^{\infty} f^{-n}(\infty)\right)$. Then there exist $R>0$ and a sequence $n_{k} \rightarrow \infty$, such that $\left|f^{n_{k}}(z)\right|<R$ for every $k$. By the definition of hyperbolicity and the fact that the spherical and Euclidean metric are comparable on compact sets in $\mathbf{C}$ we conclude that there exists $r>0$ such that $\mathcal{D}\left(f^{n_{k}}(z), r\right) \cap \overline{\mathcal{P}(f)}=\emptyset$ for every $k$, which gives $z \in J_{r}(f)$.

The proof of the following proposition, using the Lebesgue Density Theorem, Theorem 2.5 and a normality argument, is rather standard, so it is omitted.

Proposition 3.2. $\quad$ (a) If $J(f) \neq \mathbf{C}$ (which holds if and only if $J(f)$ has empty interior), then the 2-dimensional Lebesgue measure of $J_{r}(f)$ is zero.

(b) If $f$ is hyperbolic, then the 2-dimensional Lebesgue measure of $J(f) \backslash I(f)$ is zero.

The next two propositions describe properties of conformal measures. The first one, which can be proved by a straightforward computation, shows that the notion 
of $t$-conformality is independent of chosen conformal metric, at least within some range.

Proposition 3.3. Let $\nu$ be a $t$-conformal measure with respect to a conformal metric $\left|d \rho_{1}\right|$ and let $\left|d \rho_{2}\right|=\rho_{2}|d z|$ be another conformal metric. If

$$
M=\int \eta d \nu<\infty \quad \text { for } \eta(z)=\left(\frac{\rho_{2}(z)}{\rho_{1}(z)}\right)^{t},
$$

then the measure $\mu$ defined as

$$
d \mu=\frac{\eta}{M} d \nu
$$

is $t$-conformal with respect to the metric $\left|d \rho_{2}\right|$. If $M=\infty$, then the measure $\mu$ defined as $d \mu=\eta d \nu$ satisfies (4) but is infinite.

The following proposition describes an alternative concerning properties of conformal measures, which can appear for non-hyperbolic maps $f$.

Proposition 3.4. If $\nu$ is a $t$-conformal measure on $J(f)$ for some $t>0$, then either $\nu$ is positive on non-empty open sets in $J(f)$ or $\nu$ is supported on the set of (at most two) exceptional values of $f$.

Proof. Suppose $\nu(B)=0$, where $B=\mathbf{D}(z, r)$ for some $z \in J(f)$ and $r>0$. We consider separately two complementary cases. First, assume that all iterates $f^{n}$ are defined on $B$. Since $\nu(B)=0$, we have $\nu\left(f^{n}(B)\right)=0$ for every $n \geq 0$. This follows easily from the formula (4) and the fact that the disc $B$ can be divided into a finite union of Borel subsets on which the map $f^{n}$ is injective. Hence, $\nu$ is supported on $A=\mathbf{C} \backslash \bigcup_{n \geq 0} f^{n}(B)$. Since the family $\left\{\left.f^{n}\right|_{B}\right\}_{n \geq 0}$ is not normal, the set $A$ has at most two elements $p_{1}, p_{2} \in \mathrm{C}$. By definition, $f^{-1}\left(\left\{p_{1}, p_{2}\right\}\right) \subset\left\{p_{1}, p_{2}\right\}$, so $p_{1}, p_{2}$ are exceptional values of $f$.

In the second case, there exists $n \geq 0$ such that $f^{n}(B)$ contains some pole of $f$, so $f^{n+1}(B)$ contains a neighbourhood of $\infty$. By Picard's/Nevanlinna Theorem, the function $f$ assumes every value infinitely many times in every neighbourhood of $\infty$, except of at most two points $p_{1}, p_{2} \in \widehat{\mathbf{C}}$, which are exceptional values of $f$. This implies $f^{n+2}(B) \supset \widehat{\mathbf{C}} \backslash\left\{p_{1}, p_{2}\right\}$. Again, dividing $B$ into a countable union of Borel subsets on which the map $f^{n+2}$ is injective, we see that $\nu\left(f^{n+2}(B)\right)=0$, so $\nu$ is supported on $\left\{p_{1}, p_{2}\right\}$, where we can assume $p_{1}, p_{2} \in \mathbf{C}$.

In both cases we conclude that the measure $\nu$ is supported on a set of at most two exceptional values $p_{1}, p_{2} \in \mathbf{C}$, in particular $f^{-1}\left(\left\{p_{1}, p_{2}\right\}\right) \subset\left\{p_{1}, p_{2}\right\}$. Using this and (4), we easily check that one of the following possibilities must occur.

○ $f\left(p_{j}\right)=p_{j}, f^{\prime}\left(p_{j}\right)=1$ for some $j \in\{1,2\}$,

○ $f\left(p_{1}\right)=p_{2}, f\left(p_{2}\right)=p_{1}$ and $\left(f^{2}\right)^{\prime}\left(p_{1}\right)=1$,

$\circ f\left(p_{j}\right) \neq p_{j}, f^{\prime}\left(p_{j}\right)=0$ for some $j \in\{1,2\}$ (this includes the case when $p_{j}$ is a pole of $f$ of degree larger than 1).

Remark 3.5. In fact, the proof of the above proposition shows that if the Julia set of $f$ contains no parabolic cycles of length 1 or 2 and multiplier 1 composed of exceptional values of $f$, nor exceptional values which are non-fixed critical points of $f$ (including poles of $f$ of degree larger than 1 ), then every $t$-conformal measure is positive on non-empty open sets in $J(f)$.

The second alternative in Proposition 3.4 can actually occur, as noted in the following example. 
Example 3.6. For $f(z)=z e^{z}$, the value 0 is the unique finite exceptional value of $f$, with $f^{-1}(0)=\{0\}, f(0)=0$ and $f^{\prime}(0)=1$. Consequently, $0 \in J(f)$ and the Dirac measure at 0 is $t$-conformal for every $t>0$.

The next propositions consider properties of the topological pressure. The first one is an essential strengthening of [BKZ12, Proposition 5.7]. We use the notation described in (5).

Proposition 3.7. Let $f$ satisfy the assumptions of Theorem C. Then for every $t>0$, every GPS point $z_{0}$ and sufficiently large $r>0$ we have

$$
P(f, t)=\limsup _{n \rightarrow \infty} \frac{1}{n} \ln S_{n}^{\mathbf{D}(r)}\left(t, z_{0}\right) .
$$

Proof. It follows from Theorem 2.10 (see [BKZ12] for details) that

$$
P(f, t)=\sup _{r>0} \limsup _{n \rightarrow \infty} \frac{1}{n} \ln S_{n}^{\mathbf{D}(r)}\left(t, z_{0}\right)
$$

However, by Lemma 5.4 (proved in Section 5), we have

$$
S_{n}^{\mathbf{D}\left(r^{2}\right)}\left(t, z_{0}\right)=S_{n}^{\mathbf{D}\left(r^{2}\right) \backslash \mathbf{D}(r)}\left(t, z_{0}\right)+S_{n}^{\mathbf{D}(r)}\left(t, z_{0}\right) \leq c \frac{(\ln r)^{3 t}}{r^{t}} S_{n+1}^{\mathbf{D}(r)}\left(t, z_{0}\right)+S_{n}^{\mathbf{D}(r)}\left(t, z_{0}\right)
$$

for sufficiently large $r>1$ and a constant $c>0$. Hence, lim sup in the formula (6) is the same for $r$ and $r^{2}$ for large $r$. Since it is non-decreasing with $r$, this implies that it is actually constant for large $r$, which ends the proof.

The next result describes alternatives related to the existence of a zero of the pressure function.

Proposition 3.8. Let $f$ satisfy the assumptions of Theorem 2.10 and let

$$
t_{0}=\inf \{t>0: P(f, t) \leq 0\}, \quad t_{\infty}=\sup \{t \geq 0: P(f, t)=+\infty\}
$$

Then one of the following three possibilities occurs:

(a) $P\left(f, t_{\infty}\right)=\lim _{t \rightarrow t_{\infty}^{+}} P(f, t)=+\infty, t_{0}>t_{\infty}$ and $P\left(f, t_{0}\right)=0$.

(b) $0 \leq P\left(f, t_{\infty}\right)<+\infty, t_{0} \geq t_{\infty}$ and $P\left(f, t_{0}\right)=0$.

(c) $t_{0}=t_{\infty}$ and $P\left(f, t_{\infty}\right)=P\left(f, t_{0}\right)<0$.

Proof. Suppose $P\left(f, t_{\infty}\right)=+\infty$. By Theorem 2.10, $\sup _{X} P\left(\left.f\right|_{X}, t_{\infty}\right)=\infty$, where sup is taken over all compact repellers $X \subset J(f)$. Since the pressure function $P\left(\left.f\right|_{X}, t\right)$ is finite and continuous for all $t \geq 0$ (see e.g. [PU10]), this implies $\lim _{t \rightarrow t_{\infty}^{+}} P(f, t)=+\infty$. Similarly we show that if $P\left(f, t_{\infty}\right)<\infty$, then $\lim _{t \rightarrow t_{\infty}^{+}} P(f, t)=$ $P\left(f, t_{\infty}\right)$. Other assertions of the proposition follow easily from the fact that the function $t \mapsto P(f, t)$ is non-increasing and convex (and hence continuous) for $t \in$ $\left(t_{\infty},+\infty\right)$ and $P(f, 2) \leq 0$ (see Theorem 2.10).

Remark 3.9. By [BKZ09], we have $t_{0}>1$.

Note that the cases (a) and (b) in Proposition 3.8 correspond to the existence of a zero of the pressure function. It is an open problem, whether the case (c) can actually appear for a map satisfying the assumptions of Theorem 2.10. 


\section{Proof of Theorem B}

Case (a). Assume that $f \in \mathcal{S}$ and $f$ admits a $t$-conformal measure $m_{t}$ on $J(f)$ for some $t>0$, with respect to the spherical metric. Let

$$
D_{n}=\mathcal{D}\left(\bigcup_{m=0}^{n-1} f^{m}(\operatorname{Sing}(f)) \cup\{\infty\}, e^{-\sqrt{n}}\right),
$$

where we denote

$$
\mathcal{D}(X, r)=\left\{z \in \widehat{\mathbf{C}}: \operatorname{dist}_{s p h}(z, x)<r \text { for some } x \in X\right\},
$$

and

$$
E=\bigcap_{k=1}^{\infty} \bigcup_{n=k}^{\infty} D_{n}
$$

By definition, $E \subset \overline{\mathcal{P}(f)} \cup\{\infty\}$, and since $f \in \mathcal{S}$, it is easily seen that the Hausdorff dimension of $E$ equals 0 . Let

$$
A_{k}=\bigcap_{n=k}^{\infty}\left(\widehat{\mathbf{C}} \backslash D_{n}\right)
$$

for $k \geq 1$. Then $\left(A_{k}\right)_{k=1}^{\infty}$ forms an increasing sequence of sets, and

$$
\widehat{\mathbf{C}} \backslash E=\bigcup_{k=1}^{\infty} A_{k} \text {. }
$$

By the definition of $A_{k}$, the spherical area of $\widehat{\mathbf{C}} \backslash A_{k}=\bigcup_{n \geq k}^{\infty} D_{n}$ is smaller than

$$
(\# \operatorname{Sing}(f)+1) \sum_{n=k}^{\infty} n e^{-\sqrt{n}}
$$

which tends to 0 as $k \rightarrow \infty$. Hence, for large $k$ the set $A_{k}$ has positive area and, in particular, there exists a GPS point $z_{0} \in A_{k}$.

Again by the definition of $A_{k}$, for every $z \in A_{k}$ all inverse branches of $f^{n}, n \geq k$ are defined on $\mathcal{D}\left(z, e^{-\sqrt{n}}\right)$. Moreover, for every $n \geq k$ there exists at most countable partition $\left\{A_{k, j}^{(n)}\right\}_{j}$ of $A_{k}$ by non-empty Borel sets, such that $A_{k, j}^{(n)} \subset \mathcal{D}\left(v_{k, j}^{(n)}, e^{-\sqrt{n}} / 2\right)$ for some $v_{k, j}^{(n)} \in A_{k}$. By Theorem 2.5, the distortion of all inverse branches of $f^{n}$ on $A_{k, j}^{(n)}$ is bounded by a constant independent of $n$. This and the $t$-conformality of $m_{t}$ imply

$$
\frac{1}{C} m_{t}\left(A_{k, j}^{(n)}\right) S_{n}\left(t, v_{k, j}^{(n)}\right) \leq m_{t}\left(f^{-n}\left(A_{k, j}^{(n)}\right)\right) \leq C m_{t}\left(A_{k, j}^{(n)}\right) S_{n}\left(t, v_{k, j}^{(n)}\right)
$$

for some $C>0$ independent of $k, n, j$. Using the definition of $A_{k}$ and [Prz99, Lemma 3.1], we show that there exists $c>0$ such that

$$
\frac{1}{e^{c \sqrt{n}}} S_{n}\left(t, z_{0}\right) \leq S_{n}\left(t, v_{k, j}^{(n)}\right) \leq e^{c \sqrt{n}} S_{n}\left(t, z_{0}\right)
$$

for every $n \geq k$ and every set $A_{k, j}^{(n)}$ (see the proof of [BKZ12, Lemma 5.4] for details).

To prove the first assertion of the theorem, suppose that $m_{t}$ is not supported on E. Then

$$
m_{t}\left(A_{k}\right)>0
$$


for some $k$. Since $\left(A_{k}\right)_{k}$ is increasing, in fact this holds for every sufficiently large $k$. Hence, by (7) and (8), we obtain

$$
\begin{aligned}
m_{t}\left(f^{-n}\left(A_{k}\right)\right) & =\sum_{j} m_{t}\left(f^{-n}\left(A_{k, j}^{(n)}\right)\right) \geq \frac{1}{C} \sum_{j} m_{t}\left(A_{k, j}^{(n)}\right) S_{n}\left(t, v_{k, j}^{(n)}\right) \\
& \geq \frac{1}{C} \sum_{j} \frac{m_{t}\left(A_{k, j}^{(n)}\right)}{e^{c \sqrt{n}}} S_{n}\left(t, z_{0}\right)=\frac{m_{t}\left(A_{k}\right)}{C e^{c \sqrt{n}}} S_{n}\left(t, z_{0}\right) .
\end{aligned}
$$

This implies

$$
S_{n}\left(t, z_{0}\right) \leq \frac{C e^{c \sqrt{n}}}{m_{t}\left(A_{k}\right)}
$$

for every $n \geq k$, which gives $P(t, f) \leq 0$ and ends the proof of the first assertion.

To prove the second assertion, suppose $P(t, f)<0$. Then

$$
S_{n}\left(t, z_{0}\right)<e^{-n \delta}
$$

for some $\delta>0$ and every sufficiently large $n$. Using again (7) and (8), we obtain, similarly as previously, for sufficiently large $n$,

$$
\begin{aligned}
m_{t}\left(f^{-n}\left(A_{k}\right)\right) & \leq C \sum_{j} m_{t}\left(A_{k, j}^{(n)}\right) S_{n}\left(t, v_{k, j}^{(n)}\right) \leq C m_{t}\left(A_{k}\right) e^{c \sqrt{n}} S_{n}\left(t, z_{0}\right) \\
& <C e^{c \sqrt{n}-n \delta}<e^{-n \delta / 2} .
\end{aligned}
$$

This shows that for sufficiently large $k$, the series $\sum_{n} m_{t}\left(f^{-n}\left(A_{k}\right)\right)$ is convergent, so by the Borel-Cantelli Lemma, for $m_{t}$-almost every $z$ there exists $m_{0}=m_{0}(z)$ such that $f^{m}(z) \in \mathbf{C} \backslash A_{k} \subset \bigcup_{n=k}^{\infty} D_{n}$ for all $m \geq m_{0}$. This implies that for $m_{t}$-almost every point $z \in \mathbf{C}$, for every large $k$ there exists $m_{0}$ such that for every $m \geq m_{0}$ we have $\operatorname{dist}_{s p h}\left(f^{m}(z), \zeta_{m}\right)<e^{-\sqrt{k}}$ for some $\zeta_{m} \in \mathcal{P}(f) \cup\{\infty\}$. This proves the second assertion of (a).

Case (b). Assume now that $f \in \mathcal{B}$, such that $J(f) \backslash \overline{\mathcal{P}(f)} \neq \emptyset$ and $J(f)$ does not contain exceptional values of $f$. The proof is analogous to the one in case (a).

Take an open spherical disc $D=\mathcal{D}\left(z_{0}, r\right)$ for a small $r>0$, such that $\mathcal{D}\left(z_{0}, 2 r\right) \cap$ $(\overline{\mathcal{P}(f)} \cup\{\infty\})=\emptyset$. Then all inverse branches of $f^{n}, n>0$ are defined on $D$, in particular $z_{0}$ is a GPS point. By Theorem 2.5, the distortion of the branches is universally bounded. This together with the $t$-conformality of $m_{t}$ gives

$$
\frac{1}{C} m_{t}(D) S_{n}\left(t, z_{0}\right) \leq m_{t}\left(f^{-n}(D)\right) \leq C m_{t}(D) S_{n}\left(t, z_{0}\right)
$$

for some $C>0$.

Since $J(f) \backslash \overline{\mathcal{P}(f)}$ is a non-empty open set in $J(f)$ and $J(f)$ does not contain exceptional values of $f$, Proposition 3.4 implies that for every disc $D=\mathcal{D}\left(z_{0}, r\right)$ as above we have

$$
m_{t}(D)>0
$$

In particular, (9) gives

$$
S_{n}\left(t, z_{0}\right) \leq \frac{C m_{t}\left(f^{-n}(D)\right)}{m_{t}(D)}
$$

for every $n$, which implies $P(f, t) \leq 0$. This proves the first assertion.

Suppose now $P(f, t)<0$. Then there exists $\delta>0$, such that

$$
S_{n}\left(t, z_{0}\right)<e^{-n \delta}
$$


for large $n$, so (9) implies

$$
m_{t}\left(f^{-n}(D)\right)<C e^{-n \delta}
$$

for every disc $D$ as above. Again, by the Borel-Cantelli Lemma, for $m_{t}$-almost every $z$ there exists $n_{0}$ such that $f^{n}(z) \in \mathbf{C} \backslash D$ for all $n \geq n_{0}$. Since for every $\varepsilon>0$ the set $\mathbf{C} \backslash \mathcal{D}(\overline{\mathcal{P}(f)} \cup\{\infty\}, \varepsilon)$ can be covered by a finite number of discs $D$ as above, we conclude that for $m_{t}$-almost every point $z \in \mathbf{C}$ we have $f^{n}(z) \rightarrow \overline{\mathcal{P}(f)} \cup\{\infty\}$ as $n \rightarrow \infty$. This ends the proof of the second assertion of (b).

Finally, note that in the above proof, using Remark 3.5, we can obtain (10) for every $f$ such that $J(f)$ contains no parabolic cycles of length 1 or 2 and multiplier 1 composed of exceptional values of $f$, nor exceptional values which are non-fixed critical points of $f$ (including poles of $f$ of degree larger than 1 ). This proves Remark 1.2.

\section{Construction of a conformal measure - proof of Theorem C}

Throughout this section, we assume that $f$ satisfies the assumptions of Theorem $\mathrm{C}$, i.e. $f$ is a transcendental meromorphic map with a logarithmic tract over $\infty$ and $f$ is a map from class $\mathcal{S}$ or a non-exceptional map from class $\mathcal{B}$ such that $J(f) \backslash \overline{\mathcal{P}(f)} \neq \emptyset$. As noted in Remark 2.7, the maps from class $\mathcal{B}$ with at most finitely many poles have a logarithmic tract over $\infty$. Moreover, by the Iversen Theorem, exceptional values of $f$ are singular values of $f$, so hyperbolic maps are non-exceptional. Therefore, hyperbolic transcendental meromorphic maps satisfy the assumptions of Theorem C.

To prove Theorem $\mathrm{C}$, we need the following lemmas, where we fix some $t>0$. Note that the constants $c, c_{1}, c_{2}$ used in the lemmas may have different meaning depending on the context. Note also that the first two lemmas hold for arbitrary transcendental meromorphic maps $f$ with a logarithmic tract over $\infty$.

Lemma 5.1. There exist $c_{1}, c_{2}>0, r_{0}>1$ such that for every $z \in \mathbf{C}$ with $|z| \geq r_{0}$

$$
S_{1}^{\mathbf{D}\left(c_{1}(\ln |z|)^{4 \pi}\right)}(t, z)>\frac{c_{2}|z|^{t}}{(\ln |z|)^{3 t}} .
$$

Proof. By assumption, $f$ has a logarithmic tract $U$ over $\infty$. Fix $w \in \mathbf{C}$ of a large modulus $r_{0}>1$ and let $g$ be an inverse branch of $f$ defined in a neighbourhood of $w$, leading to $U$. Take an arbitrary point $z$ with $|z|>|w|$. By Lemma 2.8 applied for $z_{1}=z, z_{2}=w$, we have

$$
|g(z)|<\tilde{c}_{1}(\ln |z|)^{4 \pi}, \quad\left|g^{*}(z)\right|>\frac{\tilde{c}_{2}|z|}{(\ln |z|)^{3}}
$$

for a suitable extension of the branch $g$, where $\tilde{c}_{1}, \tilde{c}_{2}>0$ are constants depending only on $w$. Putting $c_{1}=\tilde{c}_{1}, c_{2}=\tilde{c}_{2}^{t}$ we conclude that

$$
S_{1}^{\mathbf{D}\left(c_{1}(\ln |z|)^{4 \pi}\right)}(t, z) \geq\left|g^{*}(z)\right|^{t}>\frac{c_{2}|z|^{t}}{(\ln |z|)^{3 t}} .
$$

In particular, we have the following estimate.

Corollary 5.2. There exist $c>0, r_{0}>1$ such that for every $z \in \mathbf{C}$ with $|z| \geq r_{0}$,

$$
S_{1}(t, z)>\frac{c|z|^{t}}{(\ln |z|)^{3 t}} .
$$


Lemma 5.3. Let $z_{0} \in J(f)$ be a GPS point. Then there exist $c, k_{0}>0$ such that for every $n \geq 1$ there holds

$$
S_{n+1}\left(t, z_{0}\right) \geq c \sum_{k=k_{0}}^{\infty} \frac{2^{k t}}{k^{3 t}} S_{n}^{\mathbf{D}\left(2^{k+1}\right) \backslash \mathbf{D}\left(2^{k}\right)}\left(t, z_{0}\right) .
$$

Proof. By Corollary 5.2 and the fact that the function $r \mapsto r^{t} /(\ln r)^{3 t}$ is increasing for large $r>1$, we have the following bound for sufficiently large $k$ :

$$
S_{1}(t, z)>\frac{c 2^{k t}}{(\ln 2)^{3 t} k^{3 t}} \quad \text { for } \quad z \in \mathbf{D}\left(2^{k+1}\right) \backslash \mathbf{D}\left(2^{k}\right),
$$

where $c$ is a positive constant. Therefore, for sufficiently large $k_{0}$ we obtain

$$
\begin{aligned}
S_{n+1}\left(t, z_{0}\right) & =\sum_{z \in f^{-n}\left(z_{0}\right)} \frac{S_{1}(t, z)}{\left|\left(f^{n}\right)^{*}(z)\right|^{t}} \geq \sum_{k=k_{0}}^{\infty} \sum_{\substack{z \in f^{-n}\left(z_{0}\right), z \in \mathbf{D}\left(2^{k+1}\right) \backslash \mathbf{D}\left(2^{k}\right)}} \frac{S_{1}(t, z)}{\left|\left(f^{n}\right)^{*}(z)\right|^{t}} \\
& \geq \frac{c}{(\ln 2)^{3 t}} \sum_{k=k_{0}}^{\infty} \frac{2^{k t}}{k^{3 t}} S_{n}^{\mathbf{D}\left(2^{k+1}\right) \backslash \mathbf{D}\left(2^{k}\right)}\left(t, z_{0}\right) .
\end{aligned}
$$

Using more detailed estimates provided by Lemma 5.1 instead of Corollary 5.2, we obtain also the following, slightly more delicate result, which is used in the proof of Proposition 3.7.

Lemma 5.4. Let $z_{0} \in J(f)$ be a GPS point. Then there exist $c_{1}, c_{2}>0, r_{0}>1$ such that for every $r>r_{0}$ and $n \geq 1$ there holds

$$
S_{n+1}^{\mathbf{D}\left(c_{1}(\ln r)^{4 \pi}\right)}\left(t, z_{0}\right) \geq \frac{c_{2} r^{t}}{(\ln r)^{3 t}} S_{n}^{\mathbf{D}\left(r^{2}\right) \backslash \mathbf{D}(r)}\left(t, z_{0}\right) .
$$

Proof. The proof proceeds similarly as the one of Lemma 5.3. Let $\tilde{c}_{1}=2{ }^{4 \pi} c_{1}$, where $c_{1}>0$ is the constant appearing in the formulation of Lemma 5.1. Then for every $z \in \mathbf{D}\left(r^{2}\right), r>1$, we have

$$
\mathbf{D}\left(c_{1}(\ln |z|)^{4 \pi}\right) \subset \mathbf{D}\left(\tilde{c}_{1}(\ln r)^{4 \pi}\right) .
$$

Using this together with Lemma 5.1 and the monotonicity of $r \mapsto r^{t} /(\ln r)^{3 t}$ for large $r>1$ we obtain

$$
\begin{aligned}
S_{n+1}^{\mathbf{D}\left(\tilde{c}_{1}(\ln r)^{4 \pi}\right)}\left(t, z_{0}\right) & =\sum_{z \in f^{-n}\left(z_{0}\right)} \frac{S_{1}^{\mathbf{D}\left(\tilde{c}_{1}(\ln r)^{4 \pi}\right)}(t, z)}{\left|\left(f^{n}\right)^{*}(z)\right|^{t}} \geq \sum_{\substack{z \in f^{-n}\left(z_{0}\right), z \in \mathbf{D}\left(r^{2}\right) \backslash \mathbf{D}(r)}} \frac{S_{1}^{\mathbf{D}\left(\tilde{c}_{1}(\ln r)^{4 \pi}\right)}(t, z)}{\left|\left(f^{n}\right)^{*}(z)\right|^{t}} \\
& \geq \sum_{\substack{z \in f^{-n}\left(z_{0}\right), z \in \mathbf{D}\left(r^{2}\right) \backslash \mathbf{D}(r)}} \frac{S_{1}^{\mathbf{D}\left(c_{1}(\ln |z|)^{4 \pi}\right)}(t, z)}{\left|\left(f^{n}\right)^{*}(z)\right|^{t}} \geq \frac{c_{2} r^{t}}{(\ln r)^{3 t}} S_{n}^{\mathbf{D}\left(r^{2}\right) \backslash \mathbf{D}(r)}\left(t, z_{0}\right)
\end{aligned}
$$

for sufficiently large $r>1$, where $c_{2}$ is a positive constant.

Assume that there exists $t>0$ such that $P(f, t)=0$ and fix this value of $t$ from now on. Note that we can choose a GPS point $z_{0} \in J(f)$, since the Hausdorff dimension of $J(f)$ is always positive (see [Sta94]). Since

$$
P(f, t)=\lim _{n \rightarrow \infty} \frac{1}{n} \ln S_{n}\left(t, z_{0}\right)=0,
$$


the series

$$
\sum_{n=1}^{\infty} e^{-n s} S_{n}\left(t, z_{0}\right)
$$

is convergent for $s>0$. As in [DU91c], consider now the modified sum

$$
\Sigma_{s}=\sum_{n=1}^{\infty} b_{n} e^{-n s} S_{n}\left(t, z_{0}\right)
$$

wher the sequence $b_{n}$ of positive real numbers (independent of $s$ ) can be chosen so that

$$
\lim _{n \rightarrow \infty} \frac{b_{n+1}}{b_{n}}=1, \quad \lim _{s \rightarrow 0^{+}} \Sigma_{s}=+\infty
$$

(see [DU91c, Lemma 3.1]).

As in [DU91c, MU10], we consider the probability measures $\mu_{s}, s \in(0,1)$, defined by

$$
\mu_{s}=\frac{1}{\Sigma_{s}} \sum_{n=1}^{\infty} b_{n} e^{-n s} \sum_{w \in f^{-n}\left(z_{0}\right)} \frac{\delta_{w}}{\left|\left(f^{n}\right)^{*}(w)\right|^{t}}
$$

where $\delta_{w}$ denotes the Dirac measure at $w$. In particular, the measure $\mu_{s}$ is welldefined, with its support in C. Since $z_{0} \in J(f)$, in fact $\mu_{s}$ is supported on $J(f)$.

Lemma 5.5. There exists $C>0$ such that for every $0<s<1$,

$$
\sum_{k=1}^{\infty} \frac{2^{k t}}{k^{3 t}} \mu_{s}\left(\mathbf{D}\left(2^{k+1}\right) \backslash \mathbf{D}\left(2^{k}\right)\right)<C .
$$

Proof. Write

$$
\nu_{n}=\sum_{w \in f^{-n}\left(z_{0}\right)} \frac{\delta_{w}}{\left|\left(f^{n}\right)^{*}(w)\right|^{t}}
$$

for $n \geq 1$. Then

$$
\mu_{s}=\frac{1}{\Sigma_{s}} \sum_{n=1}^{\infty} b_{n} e^{-n s} \nu_{n}
$$

By Lemma 5.3, for some $c, k_{0}>0$,

$$
\begin{aligned}
\nu_{n+1}(J(f)) & =S_{n+1}\left(t, z_{0}\right) \geq c \sum_{k=k_{0}}^{\infty} \frac{2^{k t}}{k^{3 t}} S_{n}^{\mathbf{D}\left(2^{k+1}\right) \backslash \mathbf{D}\left(2^{k}\right)}\left(t, z_{0}\right) \\
& =c \sum_{k=k_{0}}^{\infty} \frac{2^{k t}}{k^{3 t}} \nu_{n}\left(\mathbf{D}\left(2^{k+1}\right) \backslash \mathbf{D}\left(2^{k}\right)\right) .
\end{aligned}
$$

This together with (11) implies

$$
\begin{aligned}
\sum_{k=k_{0}}^{\infty} \frac{2^{k t}}{k^{3 t}} \mu_{s}\left(\mathbf{D}\left(2^{k+1}\right) \backslash \mathbf{D}\left(2^{k}\right)\right) & =\frac{1}{\Sigma_{s}} \sum_{k=k_{0}}^{\infty} \frac{2^{k t}}{k^{3 t}} \sum_{n=1}^{\infty} b_{n} e^{-n s} \nu_{n}\left(\mathbf{D}\left(2^{k+1}\right) \backslash \mathbf{D}\left(2^{k}\right)\right) \\
& \leq \frac{1}{c \Sigma_{s}} \sum_{n=1}^{\infty} b_{n} e^{-n s} \nu_{n+1}(J(f)) \\
& \leq \frac{e^{s}}{c} \sup _{m} \frac{b_{m}}{b_{m+1}} \frac{1}{\Sigma_{s}} \sum_{n=1}^{\infty} b_{n+1} e^{-(n+1) s} \nu_{n+1}(J(f))
\end{aligned}
$$




$$
\begin{aligned}
& =\frac{e^{s}}{c} \sup _{m} \frac{b_{m}}{b_{m+1}}\left(\mu_{s}(J(f))-\frac{b_{1} e^{-s}}{\Sigma_{s}} \nu_{1}(J(f))\right) \\
& <\frac{e}{c} \sup _{m} \frac{b_{m}}{b_{m+1}} \mu_{s}(J(f))=\frac{e}{c} \sup _{m} \frac{b_{m}}{b_{m+1}} .
\end{aligned}
$$

To end the proof, note that

$$
\sum_{k=1}^{k_{0}-1} \frac{2^{k t}}{k^{3 t}} \mu_{s}\left(\mathbf{D}\left(2^{k+1}\right) \backslash \mathbf{D}\left(2^{k}\right)\right) \leq \sum_{k=1}^{k_{0}-1} \frac{2^{k t}}{k^{3 t}}<\frac{2^{k_{0} t}}{2^{t}-1} .
$$

Proposition 5.6. There exists $c>0$ such that for every $0<s<1$,

$$
\sum_{k=1}^{\infty} \frac{2^{k t}}{k^{3 t}} \mu_{s}\left(J(f) \backslash \mathbf{D}\left(2^{k}\right)\right)<c .
$$

Proof. Enlarging $k_{0}$ from the previous lemmas, we can assume

$$
\frac{2^{(k-1) t}}{(k-1)^{3 t}}<q \frac{2^{k t}}{k^{3 t}}
$$

for some fixed $0<q<1$ and every $k \geq k_{0}$. Then

$$
\sum_{k=k_{0}}^{j} \frac{2^{k t}}{k^{3 t}}<\frac{1}{1-q} \frac{2^{j t}}{j^{3 t}}
$$

for every $j \geq k_{0}$. Using this and Lemma 5.5, we obtain

$$
\begin{aligned}
\sum_{k=k_{0}}^{\infty} \frac{2^{k t}}{k^{3 t}} \mu_{s}\left(J(f) \backslash \mathbf{D}\left(2^{k}\right)\right) & =\sum_{k=k_{0}}^{\infty} \sum_{j=k}^{\infty} \frac{2^{k t}}{k^{3 t}} \mu_{s}\left(\mathbf{D}\left(2^{j+1}\right) \backslash \mathbf{D}\left(2^{j}\right)\right) \\
& =\sum_{j=k_{0}}^{\infty} \sum_{k=k_{0}}^{j} \frac{2^{k t}}{k^{3 t}} \mu_{s}\left(\mathbf{D}\left(2^{j+1}\right) \backslash \mathbf{D}\left(2^{j}\right)\right) \\
& <\frac{1}{1-q} \sum_{j=k_{0}}^{\infty} \frac{2^{j t}}{j^{3 t}} \mu_{s}\left(\mathbf{D}\left(2^{j+1}\right) \backslash \mathbf{D}\left(2^{j}\right)\right)<\frac{C}{1-q}
\end{aligned}
$$

for some $C>0$ independent of $s$. Since

$$
\sum_{k=1}^{k_{0}-1} \frac{2^{k t}}{k^{3 t}} \mu_{s}\left(J(f) \backslash \mathbf{D}\left(2^{k}\right)\right) \leq \sum_{k=1}^{k_{0}-1} \frac{2^{k t}}{k^{3 t}}<\frac{2^{k_{0} t}}{2^{t}-1},
$$

this ends the proof of the proposition.

Corollary 5.7. The family $\left\{\mu_{s}\right\}_{s \in(0,1)}$ is tight. Consequently, there exists a weak limit

$$
m_{t}=\lim _{j \rightarrow \infty} \mu_{s_{j}}
$$

for some sequence $s_{j} \rightarrow 0^{+}$, which is a probability measure with support in $J(f)$.

Proof. To show the tightness of the family $\left\{\mu_{s}\right\}_{s \in(0,1)}$, it is sufficient to check that for every $\varepsilon>0$ there exists a compact subset $K$ of $J(f)$ such that $\mu_{s}(K)>1-\varepsilon$ for every $0<s<1$. This follows immediately from the estimate

$$
\mu_{s}\left(J(f) \backslash \mathbf{D}\left(2^{k}\right)\right)<c \frac{k^{3 t}}{2^{k t}}
$$


for every $0<s<1$, which is a consequence of Proposition 5.6.

Corollary 5.8. There exists a constant $c>0$ such that

$$
\sum_{k=1}^{\infty} \frac{2^{k t}}{k^{3 t}} m_{t}\left(J(f) \backslash \mathbf{D}\left(2^{k}\right)\right)<c .
$$

Proof. The estimate follows easily from Proposition 5.6 and the fact

$$
m_{t}(U) \leq \liminf _{j \rightarrow \infty} \mu_{s_{j}}(U)
$$

for every open subset $U$ of $J(f)$.

Note that Corollary 5.8 proves Remark 1.3 immediately.

Proposition 5.9. The measure $m_{t}$ is $t$-conformal with respect to the spherical metric.

Proof. The proof is almost the same as in [DU91c, MU10]. We provide it for the sake of completeness. Take a Borel set $A \subset \mathbf{C}$ such that $f$ is univalent on $A$. By definition, we have

$$
\mu_{s_{j}}(f(A))=\frac{1}{\Sigma_{s_{j}}} \sum_{n=1}^{\infty} b_{n} e^{-n s_{j}} S_{n}^{f(A)}\left(t, z_{0}\right),
$$

where $s_{j}$ is such that $\mu_{s_{j}} \rightarrow m_{t}$ as $j \rightarrow \infty$, and

$$
\begin{aligned}
\int_{A}\left|f^{*}\right|^{t} d \mu_{s_{j}} & =\frac{1}{\Sigma_{s_{j}}} \sum_{n=1}^{\infty} b_{n} e^{-n s_{j}} \sum_{w \in f^{-n}\left(z_{0}\right) \cap A} \frac{\left|f^{*}(w)\right|^{t}}{\left|\left(f^{n}\right)^{*}(w)\right|^{t}} \\
& =\frac{b_{1} e^{-s_{j}}}{\Sigma_{s_{j}}}+\frac{1}{\Sigma_{s_{j}}} \sum_{n=2}^{\infty} b_{n} e^{-n s_{j}} S_{n-1}^{f(A)}\left(t, z_{0}\right) \\
& =\frac{b_{1} e^{-s_{j}}}{\Sigma_{s_{j}}}+\frac{1}{\Sigma_{s_{j}}} \sum_{n=1}^{\infty} b_{n+1} e^{-(n+1) s_{j}} S_{n}^{f(A)}\left(t, z_{0}\right) .
\end{aligned}
$$

Take a small $\varepsilon>0$ and fix $n_{0}$ such that $\left|b_{n+1} / b_{n}-1\right|<\varepsilon$ for every $n \geq n_{0}$. Then

$$
\begin{aligned}
\left.\left|\int_{A}\right| f^{*}\right|^{t} d \mu_{s_{j}}-\mu_{s_{j}}(f(A)) \mid \leq & \frac{b_{1} e^{-s_{j}}}{\Sigma_{s_{j}}}+\frac{1}{\Sigma_{s_{j}}} \sum_{n=1}^{n_{0}-1}\left(b_{n+1}+b_{n}\right) S_{n}^{f(A)}\left(t, z_{0}\right) \\
& +\frac{1}{\Sigma_{s_{j}}} \sum_{n=n_{0}}^{\infty}\left|b_{n+1} e^{-s_{j}}-b_{n}\right| e^{-n s_{j}} S_{n}^{f(A)}\left(t, z_{0}\right) .
\end{aligned}
$$

The first and second term in the latter formula tend to 0 as $j \rightarrow \infty$, since $\Sigma_{s_{j}} \rightarrow \infty$ (see (11)). The third term can be estimated as follows:

$$
\begin{aligned}
& \frac{1}{\Sigma_{s_{j}}} \sum_{n=n_{0}}^{\infty}\left|b_{n+1} e^{-s_{j}}-b_{n}\right| e^{-n s_{j}} S_{n}^{f(A)}\left(t, z_{0}\right)=\frac{1}{\Sigma_{s_{j}}} \sum_{n=n_{0}}^{\infty}\left|e^{-s_{j}} \frac{b_{n+1}}{b_{n}}-1\right| b_{n} e^{-n s_{j}} S_{n}^{f(A)}\left(t, z_{0}\right) \\
& \leq \frac{\left(1+e^{-s_{j}}\right) \varepsilon}{\Sigma_{s_{j}}} \sum_{n=n_{0}}^{\infty} b_{n} e^{-n s_{j}} S_{n}^{f(A)}\left(t, z_{0}\right) \leq\left(1+e^{-s_{j}}\right) \varepsilon \mu_{s_{j}}(f(A)) \leq 2 \varepsilon
\end{aligned}
$$

We conclude that

$$
\left.\left|\int_{A}\right| f^{*}\right|^{t} d \mu_{s_{j}}-\mu_{s_{j}}(f(A)) \mid \rightarrow 0
$$


as $j \rightarrow \infty$. This together with the fact that

$$
m_{t}(U) \leq \liminf _{j \rightarrow \infty} \mu_{s_{j}}(U), \quad m_{t}(F) \geq \limsup _{j \rightarrow \infty} \mu_{s_{j}}(F)
$$

for every open subset $U$ and closed subset $F$ of $J(f)$ easily proves

$$
m_{t}(f(A))=\int_{A}\left|f^{*}\right|^{t} d m_{t} .
$$

The above proposition ends the proof of Theorem C.

Proof of Remark 1.4. We use Proposition 3.3 for $\nu=m_{t}, \rho_{1}(z)=1 /\left(1+|z|^{2}\right)$ and $\rho_{2}(z)=1 /\left(1+|z|^{\alpha}\right)$. To do it, we check that

$$
M=\int \eta d m_{t}=\int\left(\frac{1+|z|^{2}}{1+|z|^{\alpha}}\right)^{t} d m_{t}(z)
$$

is finite for $\alpha>1 / 2$. For $\alpha \geq 2$ this is obvious since $\eta$ is bounded in that case. For $\alpha<2$ we have

$$
\begin{aligned}
M & \leq c_{1}+c_{2} \int_{|z| \geq 1}|z|^{(2-\alpha) t} d m_{t}(z) \\
& =c_{1}+c_{2} \int_{1}^{\infty} m_{t}\left(\left\{z:|z|^{(2-\alpha) t} \geq s\right\}\right) d s \quad \text { by the Fubini Theorem } \\
& =c_{1}+c_{2}(2-\alpha) t \int_{1}^{\infty} r^{(2-\alpha) t-1} m_{t}(\mathbf{C} \backslash \mathbf{D}(r)) d r
\end{aligned}
$$

for some constants $c_{1}, c_{2}>0$. Since $t=\operatorname{dim}_{H} J_{r}(f) \leq 2$ and $\alpha>1 / 2$, we have $(2-\alpha) t-1<t$, which implies $r^{(2-\alpha) t-1}<r^{t} /(\ln r)^{3 t}$ for large $r$. Hence, by Remark 1.3, the integral in (12) is finite and Proposition 3.3 implies that the measure $\mu=m_{t}^{(\alpha)}$ is $t$-conformal with respect to the metric $\left|d \rho_{2}\right|=|d z| /\left(1+|z|^{\alpha}\right)$.

We complete the paper by the following observation considering the case $t=2$.

Proposition 5.10. Suppose $P(f, 2)=0$ and let $m_{2}$ be the 2-conformal measure constructed in the proof of Theorem C. Then the following hold.

(a) If $J(f) \neq \mathbf{C}$, then $m_{2}\left(J_{r}(f)\right)=0$.

(b) If $f$ is hyperbolic, then $m_{2}\left(J(f) \backslash\left(I(f) \cup \bigcup_{n=1}^{\infty} f^{-n}(\infty)\right)\right)=0$.

Proof. Let $\mathrm{Leb}_{2}$ denote the 2-dimensional Lebesgue measure on $\widehat{\mathbf{C}}$, with respect to the spherical metric. To prove (a), we will show

$$
\liminf _{\delta \rightarrow 0^{+}} \frac{m_{2}(\mathbf{D}(z, \delta))}{\delta^{2}}<\infty
$$

for $z \in J_{r}(f)$. This will imply that $m_{2}$ on $J_{r}(f)$ is absolutely continuous with respect to Leb $_{2}$ (see e.g. [Mat95, Theorem 2.12]) and to prove (a), it will be enough to use Proposition 3.2.

To show (13), take $z \in J_{r}(f)$ and define the sequence $n_{k}$, inverse branch of $f^{n_{k}}$, denoted by $g_{k}$, on the spherical disk $D_{k}$ and numbers $r, d_{k}$ as in the proof of Proposition 3.2. By the bounded distortion of $g_{k}$, we have

$$
\mathcal{D}\left(z, c_{1} d_{k}\right) \subset g_{k}\left(D_{k}\right)
$$

for some constant $c_{1}>0$. Moreover, $\operatorname{Leb}_{2}\left(D_{k}\right) \geq c_{2}$ for a constant $c_{2}>0$ depending only on $r$ (and thus only on $z$ ), and $m_{2}\left(D_{k}\right) \leq 1$. Recall also that $d_{k} \rightarrow 0$ as $k \rightarrow \infty$. In view of these, using the 2 -conformality of $m_{2}$ and Leb $\mathrm{b}_{2}$ together with 
the bounded distortion of $g_{k}$ and the comparability of the spherical and Euclidean metric on compact sets in $\mathbf{C}$, we obtain

$$
\frac{m_{2}\left(\mathbf{D}\left(z, c_{1} d_{k}\right)\right)}{d_{k}^{2}} \leq c_{3} \frac{m_{2}\left(g_{k}\left(D_{k}\right)\right)}{\operatorname{Leb}_{2}\left(g_{k}\left(D_{k}\right)\right)} \leq c_{4} \frac{m_{2}\left(D_{k}\right)}{\operatorname{Leb}_{2}\left(D_{k}\right)} \leq \frac{c_{4}}{c_{2}}
$$

for some constants $c_{3}, c_{4}$ independent of $k$, which shows (13) and ends the proof of (a). The assertion (b) follows immediately from (a) and Proposition 3.1.

\section{References}

[Bar95] BARAŃsKi, K.: Hausdorff dimension and measures on Julia sets of some meromorphic maps. - Fund. Math. 147:3, 1995, 239-260.

[BKZ09] Barański, K., B. Karpińska, and A. Zdunik: Hyperbolic dimension of Julia sets of meromorphic maps with logarithmic tracts. - Int. Math. Res. Not. IMRN 4, 2009, $615-624$.

[BKZ12] BARAŃski, K., B. KARPińskA, and A. ZduniK: Bowen's formula for meromorphic functions. - Ergodic Theory Dynam. Systems 32:4, 2012, 1165-1189.

[Ber93] BerGWEILER, W.: Iteration of meromorphic functions. - Bull. Amer. Math. Soc. (N.S.) 29:2, 1993, 151-188.

[Bow79] Bowen, R.: Hausdorff dimension of quasicircles. - Inst. Hautes Études Sci. Publ. Math. 50, 1979, 11-25.

[BPS01] Buzzi, J., F. PACCAut, and B. Schmitt: Conformal measures for multidimensional piecewise invertible maps. - Ergodic Theory Dynam. Systems 21:4, 2001, 1035-1049.

[CS07] Coiculescu, I., and B. Skorulski: Thermodynamic formalism of transcendental entire maps of finite singular type. - Monatsh. Math. 152:2, 2007, 105-123.

[DG99] Denker, M., and M. Gordin: Gibbs measures for fibred systems. - Adv. Math. 148:2, 1999, 161-192.

[DU91a] Denker, M., and M. URBAński: Hausdorff and conformal measures on Julia sets with a rationally indifferent periodic point. - J. London Math. Soc. (2) 43:1, 1991, 107-118.

[DU91b] Denker, M., and M. URbański: On Sullivan's conformal measures for rational maps of the Riemann sphere. - Nonlinearity 4:2, 1991, 365-384.

[DU91c] Denker, M., and M. URbański: On the existence of conformal measures. - Trans. Amer. Math. Soc. 328:2, 1991, 563-587.

[EL92] Eremenko, A. E. and Lyubich, M. Yu.: Dynamical properties of some classes of entire functions. - Ann. Inst. Fourier (Grenoble) 42:4, 1992, 989-1020.

[GS09] GraczyK, J., and S. Smirnov: Non-uniform hyperbolicity in complex dynamics. Invent. Math. 175:2, 2009, 335-415.

[KU02] Kotus, J., and M. URBański: Conformal, geometric and invariant measures for transcendental expanding functions. - Math. Ann. 324:3, 2002, 619-656.

[KU04] Kotus, J., and M. URBAŃski: Geometry and ergodic theory of non-recurrent elliptic functions. - J. Anal. Math. 93, 2004, 35-102.

[KU05] Kotus, J., and M. URbański: The dynamics and geometry of the Fatou functions. Discrete Contin. Dyn. Syst. 13:2, 2005, 291-338.

[KU06] Kotus, J., and M. URBAŃski: Geometry and dynamics of some meromorphic functions. - Math. Nachr. 279:13-14, 2006, 1565-1584.

[KU08] Kotus, J., and M. Urbański: Fractal measures and ergodic theory of transcendental meromorphic functions. - In: Transcendental dynamics and complex analysis, London Math. Soc. Lecture Note Ser. 348, Cambridge Univ. Press, Cambridge, 2008, 251-316. 
[LSV98] Liverani, C., B. Saussol, and S. Vaienti: Conformal measure and decay of correlation for covering weighted systems. - Ergodic Theory Dynam. Systems 18:6, 1998, 1399-1420.

[Mat95] Mattila, P.: Geometry of sets and measures in Euclidean spaces: fractals and rectifiability. - Cambridge Stud. Adv. Math. 44, Cambridge Univ. Press, Cambridge, 1995.

[MU96] Mauldin, R. D., and M. URbański: Dimensions and measures in infinite iterated function systems. - Proc. London Math. Soc. (3) 73:1, 1996, 105-154.

[May17] MaYer, V.: A lower bound of the hyperbolic dimension for meromorphic functions having a logarithmic Hölder tract. - Preprint, arXiv:1709.02188, 2017.

[MU10] MAYER, V., and M. URBAŃsKI: Geometric thermodynamic formalism and real analyticity for meromorphic functions of finite order. - Ergodic Theory Dynam. Systems 28:3, 2008, 915-946.

[MU10] MAYER, V., and M. URBAŃSKI: Thermodynamical formalism and multifractal analysis for meromorphic functions of finite order. - Mem. Amer. Math. Soc. 203:954, 2010.

[McM87] McMullen, C.: Area and Hausdorff dimension of Julia sets of entire functions. - Trans. Amer. Math. Soc. 300:1, 1987, 329-342.

[Pat76] Patterson, S. J.: The limit set of a Fuchsian group. - Acta Math. 136:3-4, 1976, 241273.

[Prz99] Przytycki, F.: Conical limit set and Poincaré exponent for iterations of rational functions. - Trans. Amer. Math. Soc. 351:5, 1999, 2081-2099.

[PRLS04] Przytycki, F., and J. Rivera-Letelier, and S. Smirnov: Equality of pressures for rational functions. - Ergodic Theory Dynam. Systems 24:3, 2004, 891-914.

[PU10] Przytycki, F., and M. URbański: Conformal fractals: ergodic theory methods. London Math. Soc. Lecture Note Ser. 371, Cambridge Univ. Press, Cambridge, 2010.

[Rem09] Rempe, L.: Hyperbolic dimension and radial Julia sets of transcendental functions. Proc. Amer. Math. Soc. 137:4, 2009, 1411-1420.

[Rue78] Ruelle, D.: Thermodynamic formalism: the mathematical structures of classical equilibrium statistical mechanics. - Encyclopedia Math. Appl. 5, Addison-Wesley Publishing Co., Reading, Mass., 1978.

[Sta94] Stallard, G. M.: - The Hausdorff dimension of Julia sets of meromorphic functions. J. London Math. Soc. (2) 49:2, 1994, 281-295.

[Sta99] Stallard, G. M.: The Hausdorff dimension of Julia sets of hyperbolic meromorphic functions. - Math. Proc. Cambridge Philos. Soc. 127:2, 1999, 271-288.

[Sul82] Sullivan, D.: Seminar on conformal and hyperbolic geometry. - Preprint, IHES, 1982.

[Tho12] Thomsen, K.: KMS states and conformal measures. - Comm. Math. Phys. 316:3, 2012, 615-640.

[Urb03] Urbański, M.: Measures and dimensions in conformal dynamics. - Bull. Amer. Math. Soc. (N.S.) 40:3, 2003, 281-321.

[UZ03] URbański, M., and A. ZDUnik: The finer geometry and dynamics of the hyperbolic exponential family. - Michigan Math. J. 51:2, 2003, 227-250.

[UZ04] URBański, M., and A. ZdUnik: Real analyticity of Hausdorff dimension of finer Julia sets of exponential family. - Ergodic Theory Dynam. Systems 24:1, 2004, 279-315.

[UZ07] Urbański, M., and A. Zdunik: Geometry and ergodic theory of non-hyperbolic exponential maps. - Trans. Amer. Math. Soc. 359:8, 2007, 3973-3997.

[VV10] Varandas, P., and M. Viana: Existence, uniqueness and stability of equilibrium states for non-uniformly expanding maps. - Ann. Inst. H. Poincaré Anal. Non Linéaire 27:2, $2010,555-593$. 